\title{
Oscillations of the thermal conductivity observed in the spin-liquid state of $\alpha-\mathrm{RuCl}_{3}$
}

\author{
Peter Czajka ${ }^{1, *}$, Tong Gao ${ }^{1, *}$, Max Hirschberger ${ }^{1, \dagger}$, Paula Lampen-Kelley ${ }^{2,3}$, Arnab \\ Banerjee $^{4, \ddagger}$, Jiaqiang Yan $^{3}$, David G. Mandrus ${ }^{2,3}$, Stephen E. Nagler ${ }^{4}$, and N. P. Ong ${ }^{1, \S}$ \\ ${ }^{1}$ Department of Physics, Princeton University, Princeton, NJ 08544, USA \\ ${ }^{2}$ Department of Materials Science and Engineering, \\ University of Tennessee, Knoxville, Tennessee 37996, USA \\ ${ }^{3}$ Materials Science and Technology Division, and ${ }^{4}$ Neutron Scattering Division, \\ Oak Ridge National Laboratory, Oak Ridge, Tennessee 37831, USA
}

(Dated: February 24, 2021)

In the class of materials called spin liquids, a magnetically ordered state cannot be attained even at milliKelvin temperatures because of conflicting constraints on each spin (for e.g. from geometric or exchange frustration). The resulting quantum spin-liquid (QSL) state is currently of intense interest because it exhibits novel excitations as well as wave-function entanglement. The layered insulator $\alpha-\mathbf{R u C l}_{3}$ orders as a zigzag antiferromagnet below $\sim 7 \mathrm{~K}$ in zero magnetic field. The zigzag order is destroyed when a magnetic field $\mathbf{H}$ is applied parallel to the zigzag axis a. Within the field interval $(7.3,11)$ Tesla, there is growing evidence that a QSL state exists. Here we report the observation of oscillations in its thermal conductivity below $4 \mathrm{~K}$. The oscillation amplitude is very large within the interval $(7.3,11) \mathrm{T}$ and strongly suppressed on either side. Paradoxically, the oscillations are periodic in $1 / H$, analogous to quantum oscillations in metals, even though $\alpha-\mathrm{RuCl}_{3}$ is an excellent insulator with a gap of $1.9 \mathrm{eV}$. By tilting $\mathrm{H}$ out of the plane, we find that the oscillation period is determined by the in-plane component $H_{a}$. As the temperature is raised above $0.5 \mathrm{~K}$, the oscillation amplitude decreases exponentially. The decrease anticorrelates with the emergence above $\sim 2 \mathrm{~K}$ of an anomalous planar thermal Hall conductivity measured with $\mathbf{H} \|$ a. To exclude extrinsic artifacts, we carried out several tests. The implications of the oscillations are discussed.

The Quantum Spin Liquid (QSL), first described by Anderson [1], is an exotic state of matter in which the spin wave functions are highly entangled, but long-range magnetic order is absent [2 4]. The Kitaev honeycomb model Hamiltonian $H_{K}$ for a class of spin liquids has attracted intense interest because the exact solution of its ground state in zero magnetic field features anyonic excitations that are Majorana fermions and $Z_{2}$ vortices [5].

The magnetic insulator $\alpha-\mathrm{RuCl}_{3}$ is proximate to the Kitaev honeycomb model 6 16. Interaction between the spins on $\mathrm{Ru}$ ions are described by the Kitaev exchange terms, e.g. $K_{X} \sigma_{i}^{X} \sigma_{j}^{X}$ where $X, Y$ and $Z$ define the spin axes [17, 18. Additional exchange terms $\Gamma$ and $\Gamma^{\prime}[19$ stabilize a zig-zag antiferromagnetic state below $7 \mathrm{~K}$ when the magnetic field $\mathbf{H}=0$ (Fig. 117, inset).
In a field $\mathbf{H} \| \mathbf{a}$, the zig-zag state is suppressed when $H$ exceeds the critical field $H_{C}=7.3 \mathrm{~T}$. Within the field interval $(7.3,11) \mathrm{T}$, experiments [8, 16] reveal a magnetically disordered state, identified as a quantum spin liquid (QSL) in which magnons give way to very broad modes [10, 16. Above $11 \mathrm{~T}$, the local moments are partially field-polarized. Interest was heightened by a report [20] that the thermal Hall conductivity $\kappa_{x y}$ is quantized within a 2-Kelvin window (from 3.3 to $5.5 \mathrm{~K}$ ).

Here we report measurements of both $\kappa_{x y}$ and the thermal conductivity $\kappa_{x x}$ to temperatures $T \sim 0.4 \mathrm{~K}$. We have uncovered oscillations in $\kappa_{x x}$ with amplitudes that are strongly peaked when $H$ lies in the interval $(7.3,11)$ T. Tilting $\mathbf{H}$ reveals that the oscillation period is determined by the in-plane component $H_{a}$. We note that, despite the similarity to Shubnikov de Haas (SdH) oscillations in metals, the free-carrier population at $1 \mathrm{~K}$ is exponentially suppressed by the large gap of $1.9 \mathrm{eV}$ [21].

Above $\sim 2 \mathrm{~K}$, we recorded using the stepped-field method both the thermal resistivity $\lambda_{x x}$ and thermal Hall resistivity $\lambda_{y x}$ as $H$ was slowly varied at fixed $T$ (Secs. A and B of Methods). As seen in Fig. 1b (for Sample 1 with $\mathbf{H} \| \mathbf{a})$, strong oscillations emerge in $\kappa_{x x}(H)$. Below $\sim 2 \mathrm{~K}$, the data were recorded continuously as well with the stepped-field method. The data, plotted as $\kappa_{x x} / T$ in Fig. 1k, show that the oscillation amplitudes continue to grow until they comprise $30-60 \%$ of $\kappa_{x x}$ at $0.43 \mathrm{~K}$. At $\sim 11.5 \mathrm{~T}, \kappa_{x x}$ displays a step-increase to a flat plateau. In the high-field partially polarized state, where $\kappa_{x x}$ is dominated by the phonon conductivity $\kappa^{p h}$, oscillations are rigorously absent (see below and Sec. ?? in Methods). Similar curves are observed in Sample 3 (Fig. ??a in Methods).

The oscillation amplitudes are strongly peaked in the QSL state. To extract the amplitude, we first determined the smooth background curve $\kappa_{b g}(T, H)$ threading the midpoints between adjacent extrema (Fig. ??d in Methods). The oscillatory component, defined as $\Delta \kappa=\kappa_{x x}-\kappa_{b g}$ (Fig. ??c in Methods), allowed accurate determination of the amplitude $\Delta \kappa_{a m p}$, which we plot in Fig. $1 \mathrm{~d}$ for Sample 1 (solid circles). Above $6 \mathrm{~T}, \Delta \kappa_{a m p}$ rises steeply to peak at $9.6 \mathrm{~T}$, followed by an abrupt collapse to zero above $11 \mathrm{~T}$. Below $6 \mathrm{~T}$, a weak remnant "tail" survives to $4 \mathrm{~T}$ in a mixed state in which small QSL regions coexist with the zig-zag state (we note that $4 \mathrm{~T}$ is roughly where the averaged zig-zag Bragg intensity 
begins to weaken with $H[12])$. By its profile, $\Delta \kappa_{a m p}$ is largest within the field interval $(7.3,11.5 \mathrm{~T})$ of the QSL state. The profile in Sample 3 is similar (Fig. ??a of Methods). A fourth sample 4 did not exhibit oscillations with $\mathbf{H}$ tilted at 450 to a (Table in Methods).

We next show that the oscillations are periodic in $1 / H$. Figure $2 a$ displays plots of the integer increment $\Delta n$ vs. $\left(\mu_{0} H_{n}\right)^{-1}$, where $H_{n}$ are fields locating extrema of $d \kappa_{x x} / d B$ plotted in Fig. $2 \mathrm{~b}$. We focus first on data shown as solid symbols. The data from Samples 1 (blue circles) and 3 (red stars), measured with $\mathbf{H} \| \mathbf{a}$, fall on a curve comprised of straight-line segments separated by a breakin-slope at $\sim 7 \mathrm{~T}$. The slopes $S_{f}$ of the straight segments are $41.4 \mathrm{~T}(H>7 \mathrm{~T})$ and $30.6 \mathrm{~T}(H<7 \mathrm{~T})$. When $\mathbf{H} \| \mathbf{b}$ (Sample 2, green circles), similar behavior is obtained, with the low-field slope $S_{f}$ also at $30.6 \mathrm{~T}$. However, the high-field slope is steeper with $S_{f}=64.2 \mathrm{~T}$. As shown in Figs. $1 \mathrm{~b}$ and $\mathrm{c}$, the periods are $T$ independent from 0.43 to $4.5 \mathrm{~K}$.

Taken together, the data shown in Figs. $2 \mathrm{a}$ to $2 \mathrm{~d}$ provide strong evidence that the oscillations are intrinsic and reproducible across samples. The five data sets discussed in Panel a were derived from extrema of the derivative curves $d \kappa_{x x} / d B$ displayed in Figs. 2b. The profiles show the close agreement in both period and phase between Samples 1 and 3. The matching of the extrema is especially evident in Fig. 22, which also shows that periodicity vs. $H$ (as opposed to $1 / H$ ) can be excluded. In Sample 2 the period and phase also agree with 1 and 3 for $H<7 \mathrm{~T}$ (the period is shorter above $7 \mathrm{~T}$, as already noted).

Oscillations observed with $\mathbf{H}$ tilted in the $a-c$ plane (at an angle $\theta$ to a) provide tests in an independent direction. Figure 2 shows curves of $\kappa_{x x}$ measured in Sample 1 with $\theta=0$ (blue curve), $39^{\circ}$ (purple) and $55^{\circ}$ (orange) (curves of $\kappa_{x x}$ at various $T$ are in Figs. ??a and ??b). By plotting the curves vs. $H_{a}=H \cos \theta$, we find that the periods match quite well (with a possible phase shift for the curve at $55^{\circ}$ ). The corresponding derivatives at $39^{\circ}$ and $55^{\circ}$ are plotted in Panel (b). We infer that, in tilted $\mathbf{H}$, the periods depend only on $H_{a}$. Moreover, the close matching of the blue and purple curves strongly supports an intrinsic origin.

In Sec. ?? in Methods, we discuss the relation of the oscillations to de Haas van Alphen experiments on the correlated insulator $\mathrm{SmB}_{6}$ 22]. For the proposed mechanism [23, 24] to apply to $\alpha-\mathrm{RuCl}_{3}$, we would need $H$ $\sim 10,000 \mathrm{~T}$. We also elaborate further on the evidence against artifactual origins, such as stacking faults produced by field-induced strain [25, 26].

The profile of $\Delta \kappa_{a m p}$ vs. $H$ actually imposes a tight constraint on possible mechanisms. Above $11.5 \mathrm{~T}$ in the polarized state, the oscillations vanish abruptly. Below $H_{C}$ the oscillations survive as a weak tail extending to $4 \mathrm{~T}$ in the zig-zag state. The amplitude profile suggests a close connection to the QSL state. The $1 / H$ periodicity suggests an intriguing analogy with Shubnikov de Haas oscillations, despite the absence of free carriers. We note that Landau-level oscillations have been predicted in the insulating 2D QSL state with $\mathbf{H}$ normal to the plane 27, 28. A spinon Fermi surface in the QSL state of $\alpha-\mathrm{RuCl}_{3}$ is widely anticipated 29 31. Our finding that $S_{f}$ is determined by $H_{a}$ suggests either a fully $3 \mathrm{D}$ QSL state (or possibly a different mechanism). Nonetheless, quantization of a spin Fermi surface is currently our leading interpretation.

The amplitude $\Delta \kappa_{a m p}$ is much larger in Sample 3 than in 1 (from Figs. ??a and $1 \mathrm{~d}$, the peak values are 100 and $16.5 \mathrm{~mW} / \mathrm{Km}$, respectively). We have uncovered a correlation with lattice disorder, as estimated from $\kappa^{p h}$ (see Sec. ??). In the QSL state, it is difficult to separate reliably the phonon term $\kappa^{p h}$ from the spin-excitation conductivity $\kappa_{x x}^{s}$ because of strong spin-phonon coupling (which causes oscillations in both). However, in the polarized state above $11.5 \mathrm{~T}, \kappa_{x x}$ is strongly dominated by $\kappa^{p h}$ (the profile becomes $H$ independent). Hence the plateau value of $\kappa_{x x} / T$ measures reliably the lattice disorder. At $1.0 \mathrm{~K}, \kappa_{x x} / T$ is much higher in Sample $3(2.2$ $\left.\mathrm{W} / \mathrm{mK}^{2}\right)$ than in $1\left(0.7 \mathrm{~W} / \mathrm{mK}^{2}\right)$. The lower disorder in Sample 3 correlates with a 6 -fold increase in the oscillation amplitude.

The observed status of $H_{a}$ seems empirically related to the planar thermal Hall effect (PTHE), which appears only with $\mathbf{H} \| \mathbf{a}$. At a fixed $H$, the ratio $\Delta \kappa / \kappa_{b g}$ decays with $T$ at a rate consistent with an effective mass $m^{*} \sim$ $0.64 m_{e}$ where $m_{e}$ is the free electron mass (blue circles in Fig. 33). The decay in $\Delta \kappa / \kappa_{b g}$ is accompanied by a rapid growth in the PTHE observed with $\mathbf{H} \| \mathbf{a}$ (red circles). Recently, Yokoi et al. 32] reported that $\kappa_{x y} / T$ measured with $\mathbf{H} \|$ a seems to be quantized, within a narrow interval in $T(3.8-6 \mathrm{~K})$ and and in $H\left(10<\mu_{0} H<\right.$ $11.2 \mathrm{~T}$ ). We have extended the PTHE experiment down to $T=300 \mathrm{mK}$ to gain a broader perspective.

Below $4 \mathrm{~K}$, it is necessary to use the method described in Eqs. ??-?? in Methods 33 to isolate the intrinsic thermal Hall signal $\delta_{y}$ (defined in Eq. ??) from artifacts arising from hystereses in $\kappa_{x x}$ as shown for e.g. in Fig. 35. For $\mathbf{H} \| \mathbf{b}$, the intrinsic thermal Hall signal is found to be zero for $0<H<14 \mathrm{~T}$ and $0.3<T<5 \mathrm{~K}$ (Fig. 3b, lower panel). However, with $\mathbf{H} \| \mathbf{a}$, a finite $\delta_{y}$ emerges above $\sim 2 \mathrm{~K}$, as shown in upper panel of Fig. $3 \mathrm{~b}$. The antisymmetry of $\delta_{y}$ with respect to $\mathbf{H}$ identifies it as a true PTHE. This is the thermal-conductivity analog of the true planar anomalous Hall effect observed in $\mathrm{ZrTe}_{5}$ [34.

Inverting the matrix $\lambda_{i j}(H)$ to obtain $\kappa_{i j}(H)$, we find that $\kappa_{x y}$ displays a dome profile that grows with $T$ in the QSL phase (Fig. 3d). Together, Figs. 3a and 3d provide a broad view of how the PTHE varies with $T$. While the trends of our $\kappa_{x y}$ are consistent with those in Ref. 32 (e.g. the PTHE exists only with $\mathbf{H} \| \mathbf{a}$ ), we note that the strong $T$ dependence evident in Figs. $2 \mathrm{~d}$ seems difficult to reconcile with a quantized value occuring in the interval $3.8-6 \mathrm{~K}$. Where the two data sets overlap (4-5 K), our magnitudes are much smaller $\left(\kappa_{x y} \sim 0.3\right.$ vs. $0.8 \mathrm{~mW} / \mathrm{K}^{2} \mathrm{~m}$ at $T=5 \mathrm{~K}$ ).

In summary, we have observed quantum oscillations in 
$\kappa_{x x}$ in $\alpha-\mathrm{RuCl}_{3}$ with $\mathbf{H}$ in plane. The prominence of the amplitude in the interval $(7.3,11) \mathrm{T}$ implies that they are specific to the QSL state.
[1] Anderson, P. W. Resonating valence bonds: a new kind of insulator? Mater. Res. Bull. 8, 153 (1973).

[2] Zhou, Y., Kanoda, K. \& Ng, T.K. Quantum spin liquid states, Rev. Mod. Phys. 89, 025003 (2017).

[3] Savary, L. \& Balents, L., Quantum spin liquids: a review, Rep. Prog. Phys. 80, 106502 (2017).

[4] Wen, X.G., Quantum Field Theory of Many-Body Systems, Oxford Univ. Press (2004), Ch. 9.

[5] Kitaev, A., Anyons in an exactly solved model and beyond, Ann. Phys. 321, 2-111 (2006).

[6] Plumb, K. W. et al., $\alpha-\mathrm{RuCl}_{3}$ : a spin-orbit assisted Mott Insulator on a honeycomb lattice. Phys. Rev. B 90, 041112 (2014).

[7] Sears, J. A. et al., Magnetic order in $\alpha-\mathrm{RuCl}_{3}$ : A honeycomb-lattice quantum magnet with strong spinorbit coupling. Phys. Rev. B 91, 144420 (2015).

[8] Banerjee, A. et al., Proximate Kitaev quantum spin liquid behavior in a honeycomb magnet. Nat. Mater. 15, 733-740 (2016).

[9] Sears, J. A. et al., Phase diagram of $\alpha-\mathrm{RuCl}_{3}$ in an inplane magnetic field. Phys. Rev. B 95, 180411 (2017).

[10] Wang, Z. et al., Magnetic Excitations and Continuum of a Possibly Field-Induced Quantum Spin Liquid in $\alpha$ $\mathrm{RuCl}_{3}$. Phys. Rev. Lett. 119, 227202 (2017).

[11] Leahy. I. A. et al., Anomalous thermal conductivity and magnetic torque response in the honeycomb magnet $\alpha$ $\mathrm{RuCl}_{3}$, Phys. Rev. Lett. 118, 187203 (2017).

[12] Banerjee, A. et al., Excitations in the field-induced quantum spin liquid state of $\alpha-\mathrm{RuCl}_{3}$. npj Quantum Materials (2018) 3:8 ; doi:10.1038/s41535-018-0079-2

[13] Hentrich, R. et al., Unusual phonon heat transport in $\alpha$ $\mathrm{RuCl}_{3}$ : Strong spin-phonon scattering and field-induced spin gap. Phys. Rev. Lett. 120, 117204 (2018).

[14] Lampen-Kelley , P. et al., Anisotropic susceptibilities in the honeycomb Kitaev system $\alpha-\mathrm{RuCl}_{3}$. Phys. Rev. B 98, 100403 (2018).

[15] Lampen-Kelley, P. et al., Field-induced intermediate phase in $\alpha-\mathrm{RuCl}_{3}$ : Non-coplanar order, phase diagram, and proximate spin liquid, cond-mat arXiv:1807.06192 1

[16] Balz, C. et al., Finite field regime for a quantum spin liquid in $\alpha-\mathrm{RuCl}_{3}$. Phys. Rev. B 100, 060405(R) (2019).

[17] Jackeli, G. \& Khaliullin, G., Mott Insulators in the Strong Spin-Orbit Coupling Limit: From Heisenberg to a Quantum Compass and Kitaev Models, Phys. Rev. Lett.102, 017205 (2009).

[18] Chun, S.W. et al., Direct evidence for dominant bonddirectional interactions in a honeycomb lattice iridate $\mathrm{Na}_{2} \mathrm{IrO}_{3}$, Nature Physics 11, 462 (2015).

[19] Kim, H. S. \& Kee, H. Y., Crystal structure and magnetism in $\alpha-\mathrm{RuCl}_{3}$ : An ab initio study, Phys. Rev. B 93, 155143 (2016).

[20] Kasahara, Y. et al., Majorana quantization and halfinteger thermal quantum hall effect in a Kitaev spin liquid. Nature 559, 227-231 (2018).

[21] Sinn, S. et al., Electronic Structure of the Kitaev Material $\alpha-\mathrm{RuCl}_{3}$ Probed by Photoemission and Inverse Photoemission Spectroscopies. Sci. Rep. 6, 39544; doi: 10.1038/srep39544 (2016).

[22] Tan, B.S. et al., Unconventional Fermi surface in an insulating state, Science 349, 287 (2015). DOI: 10.1126/science.aaa7974

[23] Johannes Knolle and Nigel R. Cooper, Quantum Oscillations without a Fermi Surface and the Anomalous de Haas-van Alphen Effect, Phys. Rev. Lett. 115, 146401 (2015). DOI: 10.1103/PhysRevLett.115.146401

[24] Long Zhang, Xue-Yang Song, and Fa Wang, Quantum Oscillation in Narrow-Gap Topological Insulators, Phys. Rev. Lett. 116, 046404 (2016). DOI: 10.1103/PhysRevLett.116.046404

[25] Yumi Kubota, Hidekazu Tanaka, Toshio Ono, Yasuo Narumi, and Koichi Kindo, Successive magnetic phase transitions in $\alpha$-RuCl 3 : $X Y$-like frustrated magnet on the honeycomb lattice, Phys. Rev. B 91, 094422 (2015). DOI: 10.1103/PhysRevB.91.094422

[26] H. B. Cao, A. Banerjee, J.-Q. Yan, C. A. Bridges, M. D. Lumsden, D. G. Mandrus, D. A. Tennant, B. C. Chakoumakos, and S. E. Nagler, Low-temperature crystal and magnetic structure of $\alpha-\mathrm{RuCl}_{3}$, Phys. Rev. B 93, 134423 (2016). DOI: 10.1103/PhysRevB.93.134423

[27] Motrunich, O.I., Orbital magnetic field effects in spin liquid with spinon Fermi sea: Possible application to $\kappa$ (ET) $)_{2} \mathrm{Cu}_{2}(\mathrm{CN})_{3}$, Phys. Rev. B 73, 155115 (2006). DOI: 10.1103/PhysRevB.73.155115

[28] Sodemann, I., Chowdhury, D. \& Senthil, T., Quantum oscillations in insulators with neutral Fermi surfaces, Phys. Rev. B 97, 045152 (2018). DOI: 10.1103/PhysRevB.97.045152

[29] Liu, Z. X. \& Normand, B., Dirac and Chiral Quantum Spin Liquids on the Honeycomb Lattice in a Magnetic Field, Phys. Rev. Lett. 120, 187201 (2018).

[30] Patel, N. D. \& Trivedi, N., Magnetic field-induced intermediate quantum spiin liquid with a spinon Fermi surface, PNAS 116, 12199 (2019).

[31] Takikawa, D. \& Fujimoto, S., Impact of off-diagonal exchange interactions on the Kitaev spin-liquid state of $\alpha$ $\mathrm{RuCl}_{3}$, Phys. Rev. B 99, 224409 (2019).

[32] Yokoi, T. et al., Half-integer quantized thermal Hall effect in the Kitaev material $\alpha-\mathrm{RuCl}_{3}$. cond-mat arXiv: $2001.01899 \mathrm{v} 1$.

[33] Hirschberger, M. Quasiparticle excitations with Berry Curvature in insulating magnets and Weyl semimetals (PhD thesis, Princeton University 2017).

[34] Liang, T. et al.., Anomalous Hall effect in $\mathrm{ZrTe}_{5}$, Nat. Phys. 14, 451-455 (2018). doi.org/10.1038/s41567-0180078-z 
*P.C. and T.G. contributed equally to the experiment. ${ }^{\S}$ Corresponding author email: npo@princeton.edu

${ }^{\dagger}$ Current address of M.H.: Department of Applied Physics and Quantum-Phase Electronics Center (QPEC), The University of Tokyo, Bunkyo-ku, Tokyo, 113-8656, Japan.

${ }^{\ddagger}$ A.B. is also affiliated with the Department of Physics, Purdue University, West Lafayette, IN 47907, USA.

\section{Acknowledgements}

We thank Jingjing Lin and Stephan Kim for technical assistance and T. Senthil and I. Sodemann for valuable discussions. The measurements of $\kappa_{x x}$ and P.C. and M.H. were supported by a MRSEC award from the U.S. National Science Foundation (DMR 1420541). T.G. and the low- $T$ thermal Hall experiments were supported by the U.S. Department of Energy (DE-SC0017863). A.B. and S.E.N are supported by the DOE, Office of Science, Scientific User Facilities Division. N.P.O. was supported by the Gordon and Betty Moore Foundation's EPiQS initiative through grant GBMF4539. P.L-K. and D.M. were supported by Moore Foundation's EPiQS initiative through grant GBMF4416.

\section{Author contributions}

P.C. and T.G. performed the measurements and analyzed the data together with N.P.O. who proposed the experiment. M.H. greatly enhanced the experimental technique employed. A.B., P.L-K. and S.E.N. provided guidance on prior results. Crystals were grown at ORNL by P.L-K., J.Y. and D.M. at ORNL. The manuscript was written by N.P.O., P.C. and T.G.

\section{Additional Information}

Supplementary information is available in the online version of the paper. Correspondence and requests for materials should be addressed to N.P.O. Reprints and permissions information is available at www.nature.com/reprints.

\section{Competing financial interests}

The authors declare no competing financial interests.

\section{Figure Captions}

Figure 1: Quantum oscillations in the quantum spin liquid (QSL) phase in $\alpha-\mathbf{R u C l}_{3}$ (Sample 1). Panel (a): The phase diagram showing the QSL phase sandwiched between the zig-zag and polarized states with $\mathbf{H} \| \mathbf{a}$ (axes $\mathbf{a}$ and $\mathbf{b}$ shown in inset). The ZZ2 phase lying between critical fields $B_{c 1}$ and $B_{c 2}$ is outlined by the dashed curve [16]. Panel (b) shows the emergence of oscillations in $\kappa_{x x}(H)(\mathbf{H} \| \mathbf{a})$ as $T$ falls below $4 \mathrm{~K}$. Data recorded using the stepped-field technique to correct for magnto-caloric effects (Sec. B in Methods). Panel (c) displays the oscillations over the full field range at selected $T$ (data recorded continuously as well as with steppedfield method). At $\sim 11 \mathrm{~T}, \kappa_{x x}$ displays a step-increase to a plateau-like profile in the polarized state in which oscillations are strictly absent. The amplitude $\Delta \kappa_{a m p}$ (solid circles in Panel (d)) is strikingly prominent in the QSL state. Its profile (shaded orange) distinguishes the QSL from adjacent phases. A weak remnant tail extends below $7 \mathrm{~T}$ to $4 \mathrm{~T}$ in the zig-zag state. The derivative curves $d\left(\kappa_{x x} / T\right) / d B$ show that the oscillations onset abruptly at $4 \mathrm{~T}$. The large derivative peak centered at $\sim 11.3 \mathrm{~T}$ corresponds to the step-increase in $\kappa_{x x}$ mentioned, and is not part of the oscillation sequence.

Figure 2: Periodicity and intrinsic nature of oscillations (panels labelled clockwise). Panel (a) plots the integer increment $\Delta n$ versus $1 / H_{n}$ (or $1 / H_{n, a}$ ) where $H_{n}$ are the fields identifying extrema of the derivative curves $d \kappa_{x x} / d B\left(H_{n, a}=H_{n} \cos \theta\right.$ for tilted $\left.\mathbf{H}\right)$. Solid symbols represent data taken with $\mathbf{H}$ strictly in-plane. The blue circles (Sample 1) and red stars (Sample 3) were measured with $\mathbf{H} \| \mathbf{a}$, whereas the green circles were measured in Sample 2 with $\mathbf{H} \|$ b. Open symbols are measurements in Sample 1 with $\mathbf{H}$ tilted in the $a$ $c$ plane at angles $\theta=39^{\circ}$ (triangles) and $55^{\circ}$ (circles), relative to $\mathbf{a}$. The data sets fall on the same segmented curve (comprised of line segments with slope $31 \mathrm{~T}$ below $7 \mathrm{~T}$ and $41 \mathrm{~T}$ above $7 \mathrm{~T}$ ). The exception is the high-field slope of $64 \mathrm{~T}$ in Sample 2 with $\mathbf{H} \| \mathbf{b}$. For clarity, the 3 data sets are shifted vertically by $\Delta n=1$. Panel (b): Curves of the derivative $d \kappa_{x x} / d B$ vs. $1 / H$ (or $1 / H_{a}$ ) for Samples 1, 2 and $3\left(H_{a}=H \cos \theta\right)$. For Sample 1, we show $d \kappa_{x x} / d B$ measured with $\theta=0,39^{\circ}$ and $55^{\circ}$. The extrema of $d \kappa_{x x} / d B$ are plotted in Panel (a). Vertical lines mark the values of $1 / H_{n}$ and $1 / H_{n, a}$ read off from the straight-line fits in Panel (a) for integer increments $\Delta n$. Panel (c): Replot of integer increment $\Delta n$ vs. $H_{n}$ (fields locating the extrema of $d \kappa_{x x} / d B$ ) in Samples 1 (blue circles) and 3 (red stars) to check for periodicity vs. $H$ (as opposed to $1 / H)$. In both data sets (measured with $\mathbf{H} \| \mathbf{a}$ ), the curve diverges to large negative $\Delta n$ as $H$ decreases to $4 \mathrm{~T}$. The narrowing of the spacing between adjacent extrema is strongly incompatible with periodicity vs. $H$. Panel (d) shows the effect of tilting $\mathbf{H}$ out of the plane in Sample 1 by angle $\theta$ (relative to a) at $T \sim 0.6 \mathrm{~K}$. The curves are measured with $\theta=0^{\circ}$ (blue), 
$39^{\circ}$ (purple) and $55^{\circ}$ (orange). When they are plotted vs. $H_{a}$, the periods of the oscillations in $\kappa_{x x}$ match well for the 3 angles.

Figure 3: The planar thermal Hall response. Panel (a) The $T$ dependence of $\Delta \kappa / \kappa_{b g}$ at $8.4 \mathrm{~T}$ (blue circles) and the planar thermal Hall conductivity $\kappa_{x y}$ at $9 \mathrm{~T}$ (red). The decrease of $\Delta \kappa / \kappa_{b g}$ with $T$ (consistent with an effective mass $\left.m^{*} / m_{e}=0.64\right)$ is anti-correlated with the increase in $\kappa_{x y}$. Panel (b) shows the emergence of the PTHE signal $\delta_{y}$ with $\mathbf{H} \| \mathbf{a}$ (upper panel). At $T=4.03 \mathrm{~K}, \delta_{y}$ in Sample 1 (left axis) displays sharp peaks that are antisymmetric in $H$ for $\mathbf{H} \|$ a (black cir- cles). Corresponding values of $\lambda_{y x}$ are on the right axis. The lower panel shows the null thermal Hall resistivity (expressed as the thermal Hall signal $\delta_{y}$ ) measured with $\mathbf{H} \| \mathbf{b}$ at $0.3,2.6$ and $5 \mathrm{~K}$ in Sample 2. The total uncertainty in $\delta_{y}$ is $0.3 \mathrm{mK}$. Panel (c) shows the hysteresis in $\kappa_{x x}$ that can contaminate $\kappa_{x y}$ if not properly subtracted. The right-going (purple) and left-going (red) scans have been antisymmetrized with respect to $H$. In Panel (d), $\kappa_{x y}(H)$ derived from the measured tensor $\lambda_{i j}$ are plotted for several $T$ from 3.4 to $5.5 \mathrm{~K}$. The dome-shaped profiles are the planar thermal Hall effect reported in Ref. 32 but in our experiment the values are not quantized. 
a
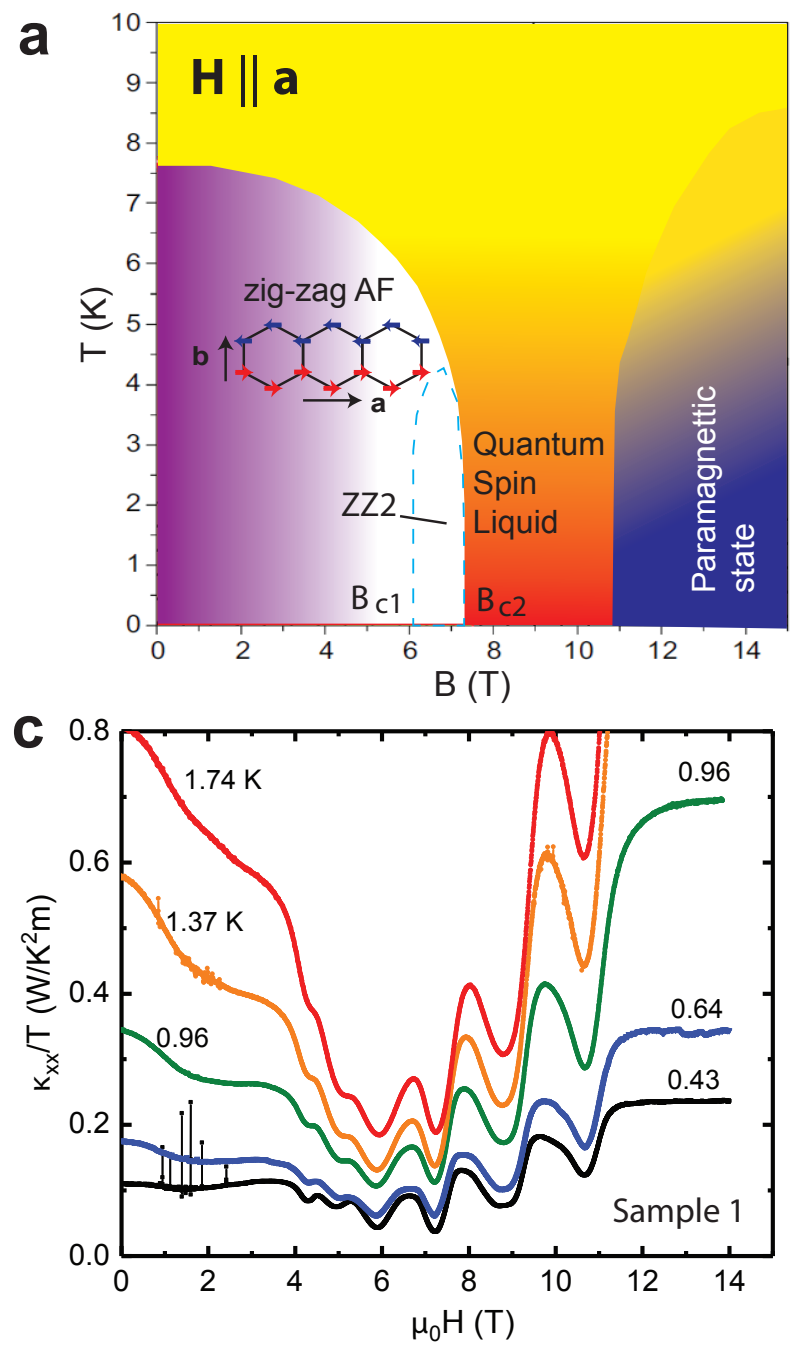
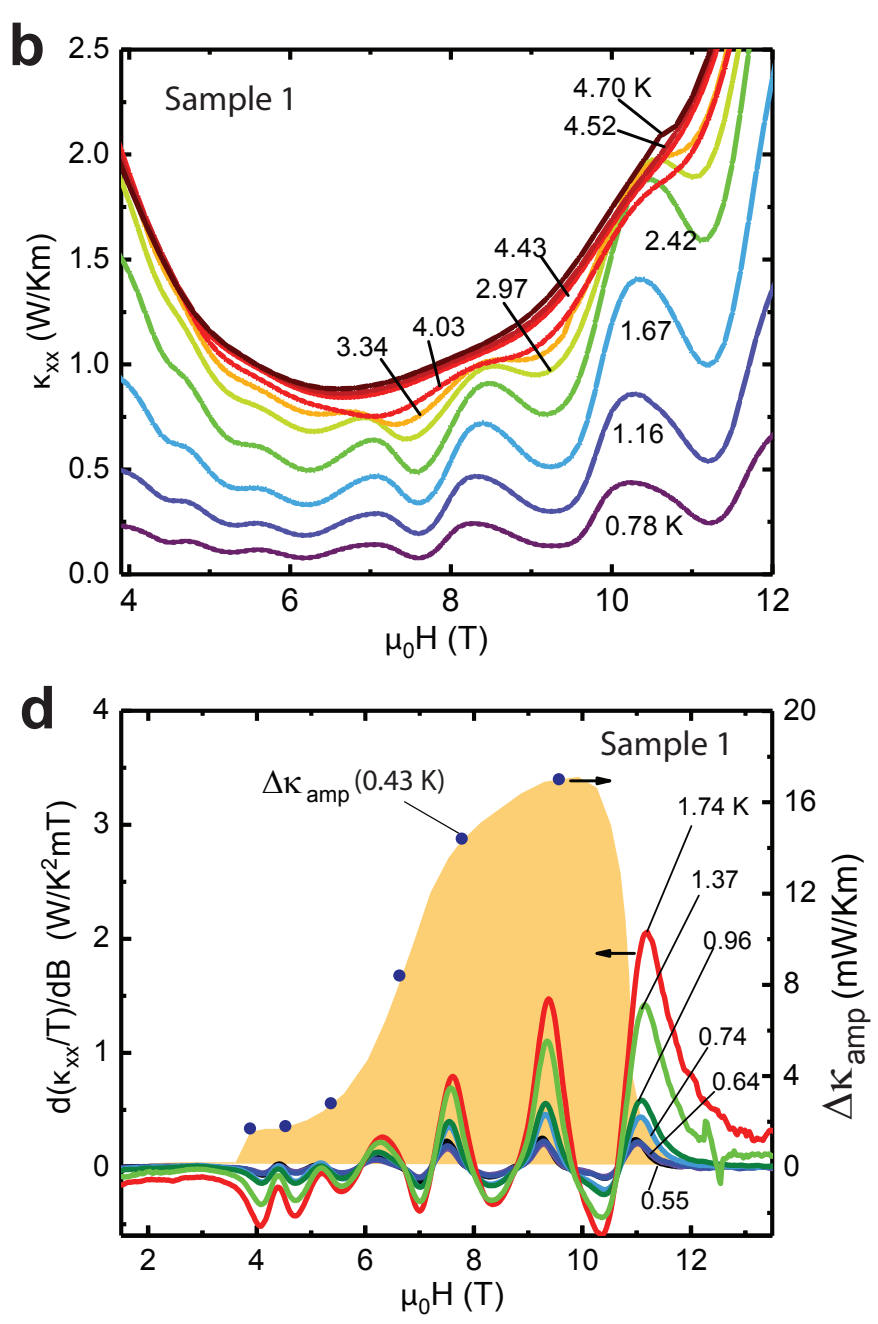

FIG. 1. 

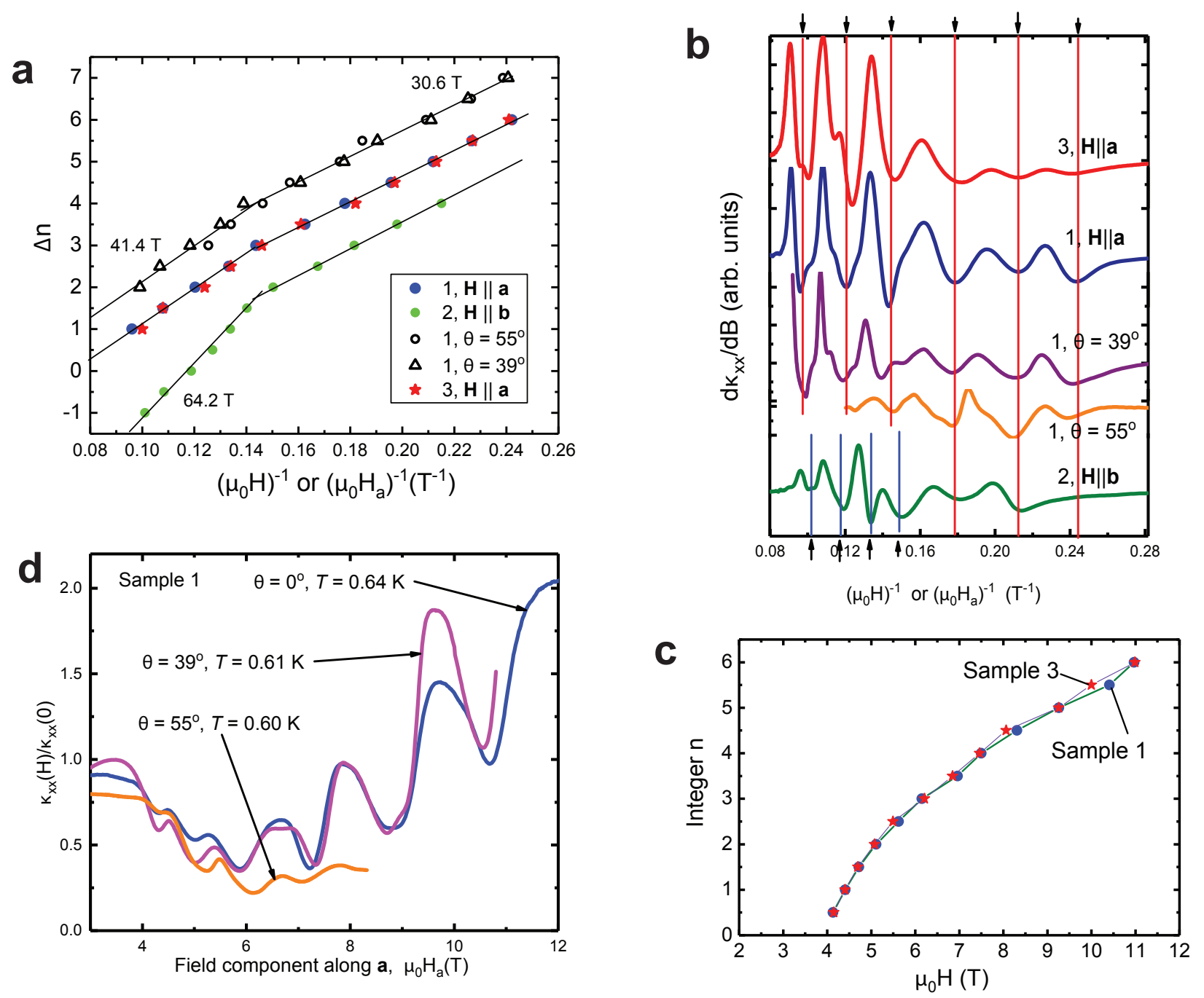

FIG. 2. 


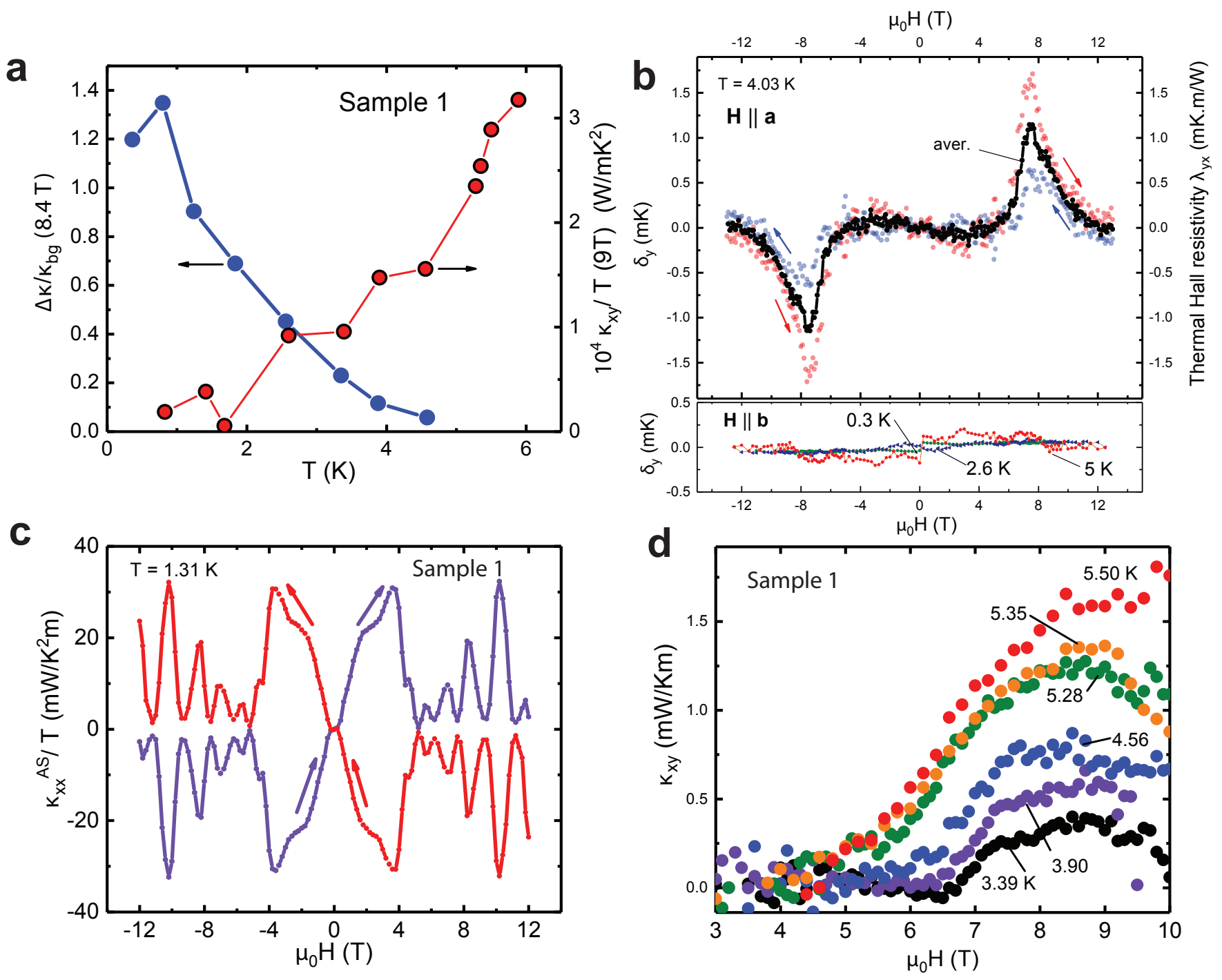

FIG. 3. 